\title{
Effects of Reciprocal Inhibition Using Thera-band on Scapular Muscle Activities During Arm-lifting Exercises in Subjects with Rounded Shoulder Posture
}

\author{
Chi-Hun Lee, PT • Heon-Seock Cynn, PT, $\mathrm{PhD}^{\dagger} \cdot \mathrm{A}-$ Reum Shin, PT, PhD $\cdot$ Ji-Hyun Lee, PT, PhD ${ }^{1}$ \\ Department of Physical Therapy, Graduate School, Yonsei University, \\ ${ }^{1}$ Department of Physical Therapy, Baekseok University
}

Received: May 19, 2020 / Revised: May 25, 2020 / Accepted: June 24, 2020

(c) 2020 J Korean Soc Phys Med

\section{| Abstract |}

PURPOSE: Excessive activity of the upper trapezius (UT) muscle contributes to a rounded shoulder posture (RSP) through abnormal rotation of the scapula that weakens the lower trapezius (LT) and serratus anterior (SA) muscles. This study compared the effects of two arm-lifting exercises with and without the use of a Thera-band on the activities of LT, SA, and UT muscles, and the LT/UT and SA/UT activity ratio in subjects with a rounded shoulder posture.

METHODS: Sixteen subjects with RSP participated in this study. All subjects performed arm-lifting (AL), diagonal arm-lifting (DAL), arm lifting with isometric adduction (ALIA), and diagonal arm-lifting with isometric adduction (DALIA) exercises. The surface electromyography data, LT, SA, UT, and the LT/UT and SA/UT activity ratios were measured. A paired t-test was used to compare the differences between two arm-lifting exercises and two arm lifting with

$\dagger$ Corresponding Author : Heon-Seock Cynn cynn@yonsei.ac.kr, https://orcid.org/0000-0002-5810-2371

This is an Open Access article distributed under the terms of the Creative Commons Attribution Non-Commercial License (http://creativecommons.org/licenses/by-nc/3.0) which permits unrestricted non-commercial use, distribution, and reproduction in any medium, provided the original work is properly cited. isometric adduction exercises.

RESULTS: In ALIA and DALIA exercises, the UT muscle activity decreased significantly, whereas the LT/UT, SA/UT activity ratio increased significantly. The activity of SA muscle increased significantly more with DALIA than that with DAL.

CONCLUSION: Arm-lifting exercises using a Thera-band can be implemented as an effective way to reduce the UT overactivity and increase the SA activity and LT/UT, SA/UT activity ratio in subjects with RSP.

Key Words: Upper trapezius, Reciprocal inhibition, Serratus anterior, Lower trapezius

\section{Introduction}

Rounded shoulder posture (RSP) is featured by downward rotation, protraction, and anterior tilting of the scapula with increased lordosis of cervical and kyphosis of upper thoracic [1-3]. Several factors, such as loss of activity of the lower trapezius (LT) and serratus anterior (SA) muscles, may cause RSP [4-6]. Weakness of the LT and SA can result in overactivation of the upper trapezius (UT) and contribute to impingement through abnormal rotation of the scapula $[4,7-9]$. 
Previous studies have reported that LT and SA muscle strengthening was included routinely in rehabilitation to prevent RSP [10-13]. Arm-lifting exercises with a scapular posterior tilt (SPT) are most effective way to strengthen LT and SA and are used to stabilize the scapula $[11,14]$. $\mathrm{Ha}$ [15] reported that backward-rocking arm-lifting exercise performed simulates maximal SPT and facilitates the activation of SA. Backward-rocking diagonal arm-lifting exercises were most effective way to activate the LT muscle in healthy subjects [14].

The effects of reciprocal inhibition using Thera-band, which increases the activation of target muscles and decreases the overactivation of the dominant muscle, were reported [16-17]. Previous researchers reported that significantly higher SA activity and lower UT and LS activities in scapular depression in addition to scapular upward rotation compared with scapular depression because of facilitating scapular depressors by reciprocally inhibiting scapular upward rotator [17]. These results were accounted for reciprocal inhibition of scapular elevators during SPT with depression in subjects with RSP and UT muscle. Park et al. reported that the use of Thera-band demonstrated higher SA activity during isometric horizontal abduction through reciprocal inhibition of the pectoralis major in subjects with winging scapula [16]. On the other hand, to the best of the authors' knowledge, no previous studies have reported strategies to decrease the overactivation of the UT and increase activation of LT and SA during arm-lifting exercises using Thera-bands in subjects with RSP.

Therefore, this study investigated the effects of reciprocal inhibition using a Thera-band on the muscle activities of LT, SA, and UT, and the LT/UT, SA/UT activity ratio during arm-lifting exercises (arm-lifting exercise versus arm-lifting with isometric adduction, the diagonal arm-lifting exercise versus diagonal arm-lifting with isometric adduction) in subjects with RSP. It was hypothesized that reciprocal inhibition using Thera-band during arm-lifting exercises would decrease the UT overactivity and increase the LT and SA muscle activity, as well as the LT/UT and SA/UT ratios among subjects with RSP.

\section{Methods}

\section{Subjects}

G*power software ver. 3.1.2 (Franz Faul, University of Kiel, Kiel, Germany) was used for statistical power analysis. A sample size of 16 was calculated from a pilot study of four subjects to achieve a power of .80 and an effect size of .69 (Calculated from the partial $\eta^{2}$ of .21 from a pilot study), with a level of .05 . Thus, 16 men with RSP participated (age $=21.69 \pm 2.09$ years, height $=174.05 \pm .04 \mathrm{~cm}$, weight $=72.00 \pm 8.37 \mathrm{~kg}$, and BMI $\left.=23.76 \pm 2.52 \mathrm{~kg} / \mathrm{m}^{2}\right)$ in the study. The RSP of both shoulders was measured using calipers by the principal investigator (PI), who had six years of clinical experience in musculoskeletal assessment and intervention. RSP was indicated by a distance $\geq 2.5 \mathrm{~cm}$ from the posterior aspect of the acromion to the table in the supine position [5]. Subjects who fulfilled the RSP criteria were recruited. In subjects who fulfilled the criteria in both shoulders, the side showing more severe RSP was adopted as the experimental arm. The inclusion criteria were as follows: 1) RSP (A score $\geq 2.5 \mathrm{~cm}$ ); 2) ability to perform the full range of motions comfortably in the sagittal, frontal, and scapular planes; 3) ability to maintain arm lifting for five seconds with the backward-rocking position. The exclusion criteria were shoulder pain or a history of surgery owing to musculoskeletal disease, which interfered with the test position. The subjects were male Yonsei University students in their twenties and were recruited through public notices. Before participation, the procedure was explained to all subjects, after which they provided written informed consent. The Yonsei University Wonju Institutional Review Board approved this study (1041849-201812-BM-129-02). 


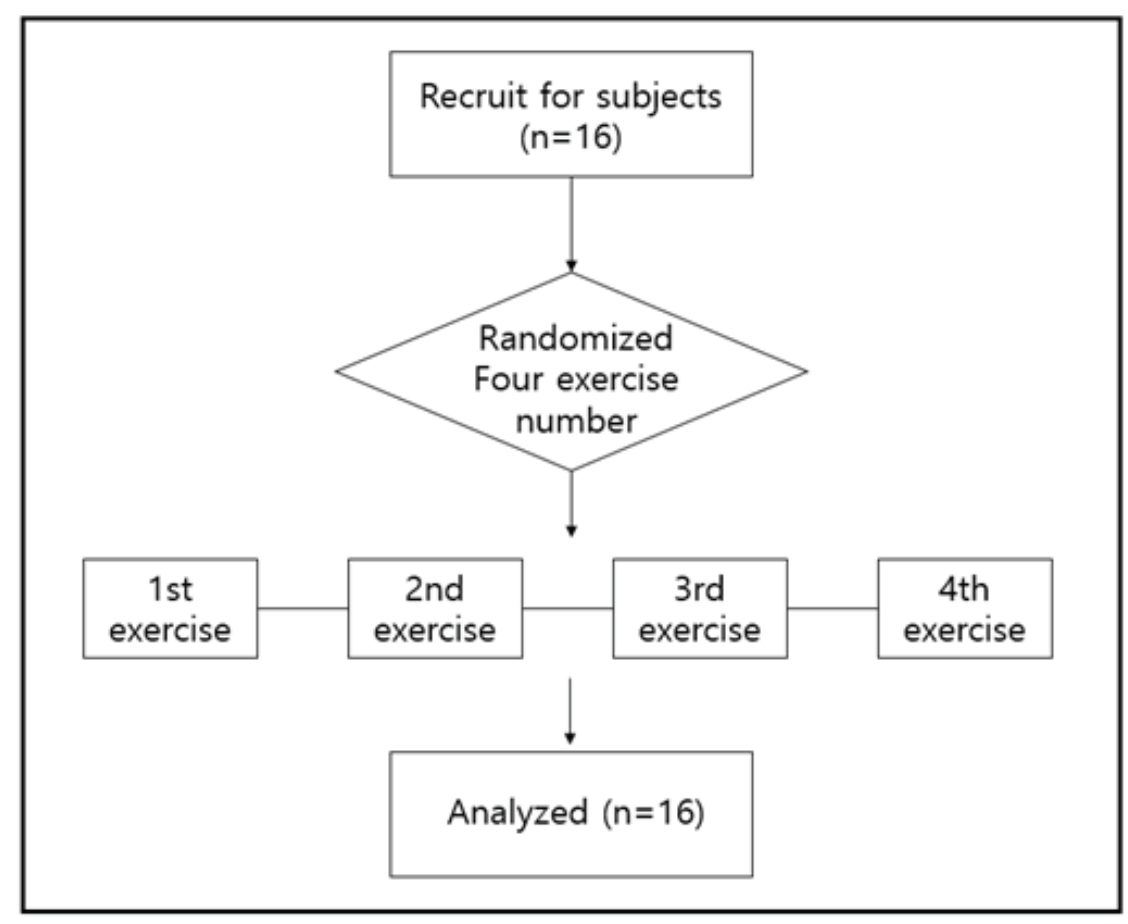

Fig. 1. Flow chart.

2. Electromyography (EMG) recording and data processing

EMG data were collected using a wireless TeleMyo DTS (Noraxon Inc., Scottsdale, AZ, USA), and the Myo-Research Master Edition 1.06 XP software (Noraxon Inc., Scottsdale, AZ, USA) was used to analyze the EMG data. The EMG signals were sampled at 1,000 $\mathrm{Hz}$. A bandpass filter between 20 and $450 \mathrm{~Hz}$ was used, and a notch filter was preset to reject $60 \mathrm{~Hz}$. The raw data were processed into the root mean square with a window of $50 \mathrm{~ms}$. Two surface electrodes with an inter-electrode distance of $2 \mathrm{~cm}$ were positioned on the UT, LT, and SA muscles. Two electrodes were placed in the middle of each muscle belly and parallel to the muscle fibers. The electrode sites were shaved, and rubbing alcohol was used to reduce the skin impedance [18]. The UT muscle electrodes were attached at the midpoint of the muscle belly between the $\mathrm{C} 7$ spinous process and the acromioclavicular joint, which is the insertion point of the trapezius muscle [19]. The LT muscle electrodes were attached at an oblique vertical angle; other electrodes were placed superior and inferior to a point 5 $\mathrm{cm}$ inferomedial from the root of the spine of the scapula. The SA muscle electrodes were attached vertically along the mid-axillary line at the 6th-8th rib [20]. Correct electrode placement was confirmed by a visual inspection of the EMG signals on a computer screen during specific muscle testing. Maximal voluntary isometric contractions (MVIC) were collected to normalize the EMG data from the UT, LT, and SA muscles using the manual muscle testing positions recommended by previous studies [21-22]. To obtain the MVIC value for UT, the subjects were seated on the treatment table with no back support. The patient's arm was abducted to $90^{\circ}$, and the head was flexed laterally $10^{\circ}-15^{\circ}$ towards the abducted arm. The subjects were then instructed to attempt approximating the abducted arm and head toward each other against the examiner's matching 
immovable resistance [23]. To determine the MVIC value for LT, the subjects were tested in the prone position. The patient's arm was placed diagonally overhead, in line with the lower fibers of the trapezius muscle during external rotation, while a resistance was applied just above the elbow joints [11,22]. To obtain the MVIC value for SA, the subjects were seated on the treatment table with no back support. The patient's shoulder was rotated internally and abducted to $125^{\circ}$ in the scapular plane, while a resistance was applied above the elbow by the PI [21]. Each contraction was held for 5 seconds, with the maximal effort against manual resistance, and a 2-minute rest was provided between trials to minimize muscle fatigue [24]. The first and last seconds of the EMG data from each MVIC trial were discarded, and the remaining three seconds of data were used [25-26]. The mean value of three seconds in three trials was taken, and the mean of the three trials was calculated for data analysis. The intra-class correlation coefficient (ICC) for MVICs of UT, LT, and SA were .96 (95\% Confidence Interval [CI]: .87-.99) and .94 (95\% CI: .83-.98), respectively. The EMG amplitudes for UT, LT, and SA during the exercises were expressed as a percentage of the mean MVIC (\%MVIC).

\section{Procedures}

The PI instructed subjects uniformly regarding the standardized position of the four arm-lifting exercises and how to perform each exercise. The subjects were then allowed to familiarize themselves with the exercise for approximately 30-minutes until the proper motion was achieved. The order of the recording of the four exercises was randomized using the random number generator in Microsoft Excel (Microsoft Corp., Redmond, WA, USA). For data collection, the subjects performed three trials of each exercise with a one-minute rest period between the trials. Each of the four arm-lifting exercises was performed for six seconds; the first and last seconds of each exercise trial were discarded, and the remaining four seconds of the EMG data were used [25-26]. The mean value of the three trials of each exercise was used for data analysis. A 10-minute rest period was provided between exercises to avoid fatigue. The subjects were instructed to perform arm-lifting first, followed by an isometric adduction exercise. The two arm-lifting exercises with isometric adduction were similar to the exercises without isometric adduction except that the Thera-band was applied around the distal wrists to provide resistance to shoulder adduction. The tensile load of the Thera-band was determined when the subjects could perform more than 10 repetitions while maintaining a consistent metronome speed [27].

\section{Arm-lifting (AL)}

The subjects were placed in the quadruped position and instructed to rock backward slowly until their buttocks touched both heels. The subjects were asked to place the non-experimental arm under the forehead and push the forehead slightly with the dorsum of the hand to stabilize the neck and thoracic spine. The PI abducted the subject's experimental arm up to $180^{\circ}$ and confirmed the degree using a goniometer. The subject was then instructed to lift the experimental arm until the radial border of the wrist touched slightly without pushing the target bar and maintained the arm position, which was located next to the ear. A vertical pole and a height-adjustable horizontal bar were used to determine the angle of shoulder flexion (Fig. 2).

\section{Arm-lifting with Isometric Adduction (ALIA)}

ALIA was performed in an identical standardized position to AL, except that the Thera-band was used to inhibit the overactive UT reciprocally. A second vertical pole was used to tie the Thera-band, and each subject was instructed to adduct the experimental arm isometrically while performing $\mathrm{AL}$ (Fig. 3). 


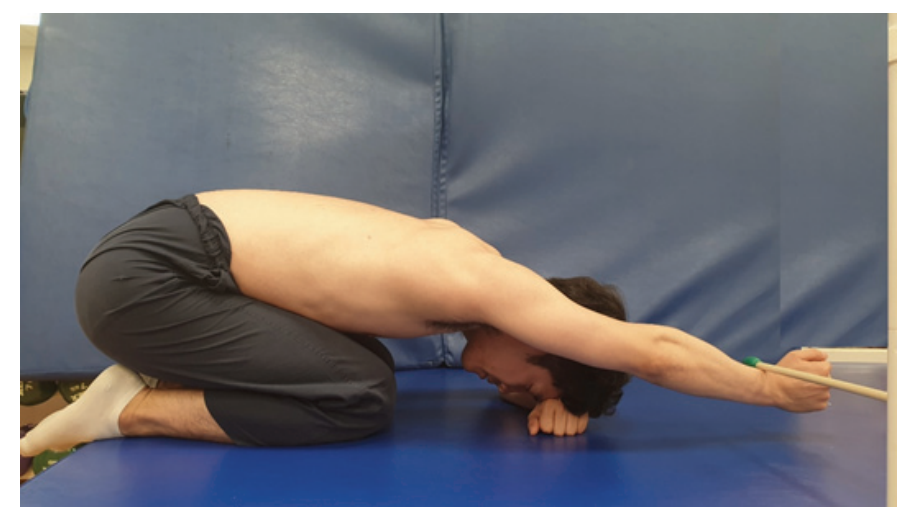

Fig. 2. Arm lifting exercise.

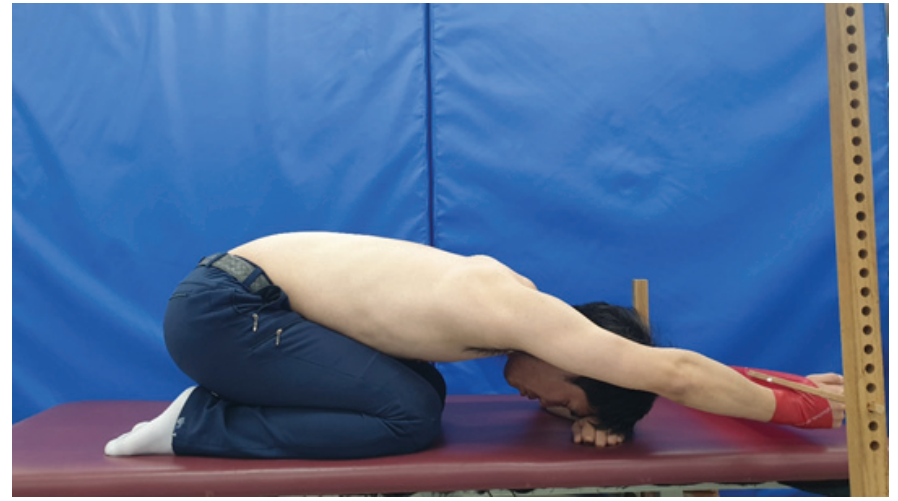

Fig. 3. Arm lifting with isometric adduction exercise.

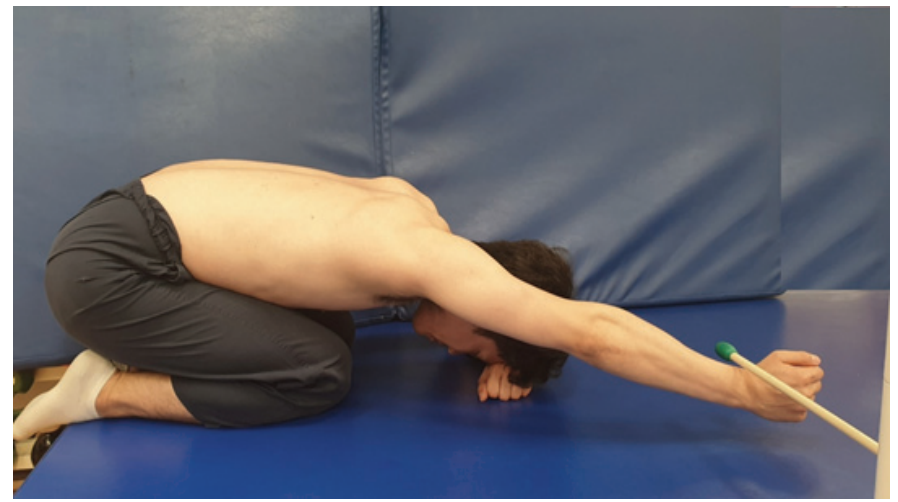

Fig. 4. Diagonal arm lifting exercise.

\section{Diagonal Arm-lifting (DAL)}

DAL was performed in an identical standardized position to $\mathrm{AL}$, except that the shoulder was abducted to $145^{\circ}$ (Fig. 4).
7. Diagonal Arm Lifting with Isometric Adduction (DALIA)

DALIA was performed in an identical standardized 


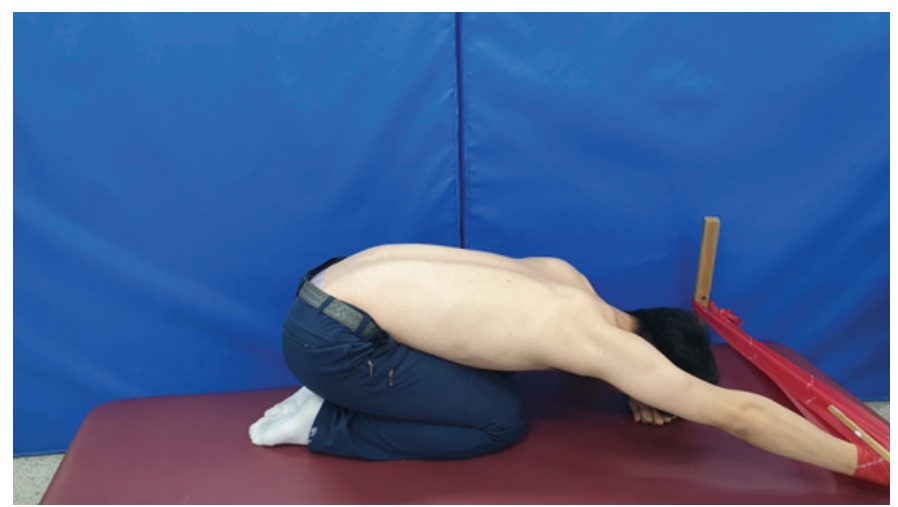

Fig. 5. Diagonal arm lifting with isometric adduction exercise.

Table 1. Comparison of the Muscle Activity and Ratio During Arm-lifting Exercise and Arm-lifting Isometric Adduction Exercise

\begin{tabular}{ccccc}
\hline Muscle or Ratio & $\begin{array}{c}\text { AL } \\
(\text { mean } \pm \text { SD) }\end{array}$ & $\begin{array}{c}\text { ALIA } \\
(\text { mean } \pm \text { SD) }\end{array}$ & t & p \\
\hline LT & $40.33 \pm 15.79$ & $46.45 \pm 25.13$ & -1.643 & .121 \\
SA & $31.70 \pm 23.90$ & $25.74 \pm 13.76$ & 1.577 & .136 \\
UT & $35.14 \pm 19.28$ & $14.44 \pm 7.93$ & 4.582 & $.000^{*}$ \\
LT/UT Ratio & $1.61 \pm 1.31$ & $3.88 \pm 2.35$ & -4.898 & $.000^{*}$ \\
SA/UT Ratio & $1.09 \pm .72$ & $2.34 \pm 1.62$ & -3.412 & $.004^{*}$ \\
\hline
\end{tabular}

Abbreviations: LT, Lower trapezius; SA, Serratus anterior; UT, Upper trapezius; AL, Arm-lifting; ALIA, Arm-lifting isometric adduction; electromyography activity is presented as a percentage of maximal voluntary isometric contraction.

position to DAL, except that the Thera-band was used to inhibit the overactive UT reciprocally. A second vertical pole was used to tie the Thera-band, and each subject was instructed to adduct the experimental arm isometrically while performing DAL (Fig. 5).

\section{Statistical Analysis}

The Kolmogorov-Smirnov Z-test was used to assess the normal distribution. Because a normal distribution was satisfied, a paired t-test was used to compare the effect of the two arm-lifting exercises and two diagonal arm-lifting exercises with isometric adduction on the activities of LT, SA, and UT, and LT/UT, SA/UT activity ratios in subjects with RSP. Statistical significance was set to .05. All statistical analyses were performed using SPSS 25 (SPSS Inc., Chicago, IL, USA).

\section{Results}

\section{LT, SA, and UT Muscle Activity}

The activity of UT muscle decreased significantly during ALIA compared to AL and during DALIA compared to DAL $(\mathrm{t}=4.582, \mathrm{p}=.001 ; \mathrm{t}=3.683, \mathrm{p}=.002$, respectively). On the other hand, the activity of SA muscle increased significantly during DALIA compared to DAL $(\mathrm{t}=-3.114$, $\mathrm{p}=.007)($ Table 1, 2).

\section{LT/UT and SA/UT Activity Ratio}

Both the LT/UT activity and SA/UT ratio increased significantly during ALIA compared with AL and during DALIA compared with DAL $(\mathrm{t}=-4.898, \mathrm{p}=.001 ; \mathrm{t}=$ $-3.746, \mathrm{p}=.002$, respectively $),(\mathrm{t}=-3.412, \mathrm{p}=.004 ; \mathrm{t}$ $=-4.327, \mathrm{p}=.001$, respectively) (Table 1,2 ). 
Table 2. Comparison of the Muscle Activity and Ratio During Diagonal Arm Lifting Exercise and Diagonal Arm-lifting Isometric Adduction Exercise

\begin{tabular}{|c|c|c|c|c|}
\hline Muscle or Ratio & $\begin{array}{c}\mathrm{DAL} \\
(\text { mean } \pm \mathrm{SD})\end{array}$ & $\begin{array}{c}\text { DALIA } \\
(\text { mean } \pm \mathrm{SD})\end{array}$ & $\mathrm{t}$ & $\mathrm{p}$ \\
\hline LT & $51.50 \pm 18.71$ & $50.41 \pm 21.31$ & .327 & .748 \\
\hline SA & $20.67 \pm 13.03$ & $26.89 \pm 14.74$ & -3.114 & $.007^{*}$ \\
\hline UT & $18.18 \pm 7.29$ & $11.10 \pm 4.20$ & 3.683 & $.002^{*}$ \\
\hline $\mathrm{LT} / \mathrm{UT}$ ratio & $3.29 \pm 1.59$ & $4.79 \pm 1.94$ & -3.746 & $.002^{*}$ \\
\hline $\mathrm{SA} / \mathrm{UT}$ ratio & $1.32 \pm .88$ & $2.62 \pm 1.52$ & -4.327 & $.001^{*}$ \\
\hline
\end{tabular}

Abbreviations: LT, Lower trapezius; SA, Serratus anterior; UT, Upper trapezius; DAL, Diagonal arm-lifting; DALIA, Diagonal arm-lifting isometric adduction; electromyography activity is presented as a percentage of maximal voluntary isometric contraction.

\section{Discussion}

The effects of two arm-lifting exercises and two armlifting exercises were compared using the Thera-band on muscle activities of LT, SA, and UT, as well as the LT/UT and SA/UT ratios in subjects with RSP. The SA muscle activity increased significantly during DALIA compared with DAL. In contrast, the UT muscle activity decreased significantly with ALIA and DALIA compared to AL and DAL, respectively. Both the LT/UT activity and SA/UT ratio increased significantly during ALIA and DALIA than that during $\mathrm{AL}$ and $\mathrm{DAL}$, respectively.

The activity of SA muscle increased significantly with DALIA than that with DAL (by 30\%), it supports hypothesis of the research. These findings are consistent with the results of various types of previous research of arm-lifting exercises to strengthen the SA and LT, treat shoulder dysfunction, such as impingement syndrome and stabilize the scapula $[6,11,14$,$] . The activity of SA muscle$ was optimal above $90^{\circ}$ shoulder elevation and a greater increase in shoulder elevation indicated more activity of SA muscle [11,21,28]. Ekstrom [11] reported that the activity of SA muscle was greater above $120^{\circ}$ of shoulder elevation in the scapular plane with healthy subjects than that below $80^{\circ}$. Hardwick [28] reported that the SA muscle was more active at $140^{\circ}$ of humeral elevation than that at $90^{\circ}$ and $120^{\circ}$ in the scapular plane with healthy subjects. Moreover, SA activates upward rotation of scapular, protraction, depression, and abduction, ultimately holding the scapula to the thoracic wall $[11,29,30]$. In the present study, the application of the Thera-band while performing AL did not show an increase in SA activity. On the other hand, it increased the SA activity during DALIA. This finding can be attributed to the efforts to stabilize the scapula during DALIA. DAL exercise is considered to be more challenging to perform than $\mathrm{AL}$. The moment arm of the DAL is longer than that of AL. Therefore, performing DAL is mechanically disadvantageous to stabilizing the scapula. Significantly higher SA activity could be considered to be a need to stabilize the scapula, especially as another scapular upward rotator (UT) was reduced by the effects of reciprocal inhibition. Therefore, DALIA is more effective than DAL in activating SA in subjects with RSP.

The UT muscle activity decreased by $59 \%$ during ALIA and by $39 \%$ during DALIA than that with $\mathrm{AL}$ and DAL, respectively. These findings support hypothesis of the research, and are consistent with previous studies, even though the application methods and materials of the Thera-band differed slightly among studies [17]. Choi and Cynn [17] reported that SPT exercise with scapular depression significantly decreased UT activities in subjects with RSP. Although various SPT exercises have been 
performed in many studies to increase the SA and LT activities $[6,14,28]$, no study has investigated the decrease in UT muscle activity caused by the application of the Thera-band (i.e., reciprocal inhibition) while performing AL exercise in subjects with RSP [31]. In this study, when the subject was instructed to perform AL exercise while maintaining isometric adduction overcoming the tension of the Thera-band, UT was inhibited reciprocally by activating the adductors and downward rotators of the experimental arm. Considering the results, isometric adduction exercise should be included to decrease the UT muscle activity during $\mathrm{AL}$ and DAL in subjects with RSP.

The activity ratio of LT/UT was significantly increased with ALIA than with AL (by 140\%) and with DALIA than with DAL (by $46 \%$ ). These results support the research hypothesis. On the other hand, no study has reported LT/UT in relation to the AL and ALIA interventions used in this study. Although there were no significant differences in the activity of LT muscle between ALIA and AL, and between DALIA and DAL, the reasons for the significant difference in the ratio were as follows. RSP is related with upper crossed syndrome (UCS), and scapular elevation observed in the UCS facilitates the UT [32]. Ludewig and Braman [33] reported the synergistic dominance of the UT over insufficient SA. They showed that an inhibited LT during scapular upward rotation leads to subacromial impingement, and overuse shoulder injuries. The ALIA and DALIA exercise inhibited the dominance of the UT. As mentioned above, reciprocal inhibition reduced the activity of UT muscle and increased the activity ratio of LT/UT. Therefore, ALIA and DALIA were more effective on the LT/UT activity ratio than AL and DAL in subjects with RSP.

SA/UT activity ratio increased significantly with ALIA and DALIA than that with AL (by 115\%) and DAL (by 99\%), respectively. These results support the research hypothesis. As mentioned above, the ALIA and DALIA exercises inhibited the dominance of the UT and simultaneously increased the muscle activity of insufficient SA and inhibited LT. Furthermore, the significantly increased SA activity could be considered as a need to stabilize the scapula. In particular, another upward scapular rotator (UT) was reduced by the effect of reciprocal inhibition. Thus, ALIA and DALIA were more effective on the SA/UT activity ratio than AL and DAL in subjects with RSP.

Several limitations of this research should be noted. First, the possibility of generalization is limited because RSP in young males was assessed. The results might be different if female subjects were included. Hence, future studies will be needed to determine if the findings of present can be generalized to symptomatic populations. Second, this study measured RSP after the each condition of SPT because kinematic data such as RSP was difficult to collect at end-range of the SPT. Further studies need to determine the correlation of scapular movement between the SPT and other end-range exercises. Finally, this study showed only cross-sectional results of muscle activities and kinematics in various conditions, so long-term effect should be considered in future studies.

\section{Conclusions}

This study investigated the effects of reciprocal inhibition using a Thera-band on the muscle activities of UT, LT, and SA, as well as the LT/UT and SA/UT activity ratios during two arm-lifting exercises in subjects with RSP. In the ALIA and DALIA exercises, the UT muscle activity decreased significantly, and the LT/UT and SA/UT activity ratio increased significantly. A significant increase in SA muscle activity was observed with DALIA compared to DAL. These findings are insightful because the study considered the potential risk from overactivation of the UT. Therefore, arm-lifting exercises using the Thera-band can be implemented as an effective way to reduce the UT overactivity and to increase the SA activity, and LT/UT 
and SA/UT activity ratio in subjects with RSP.

\section{References}

[1] Chansirinukor W, Wilson D, Grimmer K, et al. Effects of backpacks on students: Measurement of cervical and shoulder posture. Australian Journal of Physiotherapy. 2001;47(2):110-6.

[2] Wong CK, Coleman D, diPersia V, et al. The effects of manual treatment on rounded-shoulder posture, and associated muscle strength. J Bodyw Mov Ther. 2010; 14(4):326-33.

[3] Magee DJ. Orthopedic physical assessment. Elsevier Health Sciences. 2013.

[4] Ludewig PM, Cook TM. Alterations in shoulder kinematics and associated muscle activity in people with symptoms of shoulder impingement. Physical therapy. 2000;80(3):276-91.

[5] Sahrmann S. Diagnosis and treatment of movement impairment syndromes. Elsevier Health Sciences. 2002.

[6] Lee JH, Cynn HS, Yoon TL, et al. The effect of scapular posterior tilt exercise, pectoralis minor stretching, and shoulder brace on scapular alignment and muscles activity in subjects with round-shoulder posture. J Electromyogr Kinesiol. 2015;25(1):107-14.

[7] Glousman R, Jobe F, Tibone J, et al. Dynamic electromyographic analysis of the throwing shoulder with glenohumeral instability. J Bone Joint Surg Am. 1988; 70(2):220-6.

[8] McQuade KJ, Dawson J, Smidt GL. Scapulothoracic muscle fatigue associated with alterations in scapulohumeral rhythm kinematics during maximum resistive shoulder elevation. J Orthop Sports Phys Ther. 1998;28(2):74-80.

[9] Cools AM, Witvrouw EE, Declercq GA, et al. Scapular muscle recruitment patterns: trapezius muscle latency with and without impingement symptoms. Am J Sports Med. 2003;31(4):542-9.

[10] Smith J, Kotajarvi BR, Padgett DJ, et al. Effect of scapular protraction and retraction on isometric shoulder elevation strength. Archives of Physical Medicine and Rehabilitation. 2002;83(3):367-70.

[11] Ekstrom RA, Donatelli RA, Soderberg GL. Surface electromyographic analysis of exercises for the trapezius and serratus anterior muscles. Journal of Orthopaedic \& Sports Physical Therapy. 2003;33(5):247-58.

[12] Ludewig PM, Hoff MS, Osowski EE, et al. Relative balance of serratus anterior and upper trapezius muscle activity during push-up exercises. The American Journal of Sports Medicine. 2004;32(2):484-93.

[13] Hall CM, Brody LT. Therapeutic exercise: moving toward function. Lippincott Williams \& Wilkins. 2005.

[14] Ha S-m, Kwon O-y, Cynn H-s, et al. Comparison of electromyographic activity of the lower trapezius and serratus anterior muscle in different arm-lifting scapular posterior tilt exercises. Physical Therapy in Sport. 2012; 13(4):227-32.

[15] McClure PW, Bialker J, Neff N, et al. Shoulder function and 3-dimensional kinematics in people with shoulder impingement syndrome before and after a 6-week exercise program. Physical Therapy. 2004;84(9):832-48.

[16] Park KM, Cynn HS, Yi CH, et al. Effect of isometric horizontal abduction on pectoralis major and serratus anterior EMG activity during three exercises in subjects with scapular winging. J Electromyogr Kinesiol. 2013; 23(2):462-8

[17] Choi S-a, Cynn H-s, Shin A-r, et al. Effects of Verbal Cue for Scapular Depression During Scapular Posterior Tilt Exercise on Scapular Muscle Activities and Clavicular Tilt Angle in Subjects With Rounded Shoulder Posture and Upper Trapezius Myofascial Pain. Physical Therapy Korea. 2017;24(3):30-9.

[18] Cram J, Jantos M, Durie M, et al. Cram's Introduction to Surface Electromyography. 2nd. Sudbury, Massachusetts: Jones \& Bartlett Publishers. 2011.

[19] Lehman GJ, Gilas D, Patel U. An unstable support surface does not increase scapulothoracic stabilizing muscle 
activity during push up and push up plus exercises. Manual Therapy. 2008;13(6):500-6.

[20] Cram J, Kasman G, Holtz J. Introduction to surface electromyography, Aspen, 1998. ISBN 0-8342-0751-6. 1998.

[21] Ekstrom RA, Soderberg GL, Donatelli RA. Normalization procedures using maximum voluntary isometric contractions for the serratus anterior and trapezius muscles during surface EMG analysis. Journal of Electromyography and Kinesiology. 2005;15(4):418-28.

[22] Kendall FP, McCreary EK, Provance PG, et al. Muscles: Testing and Function, with Posture and Pain (Kendall, Muscles). Philadelphia: Lippincott Williams \& Wilkins. 2005.

[23] Schüldt K, Harms-Ringdahl K. Activity levels during isometric test contractions of neck and shoulder muscles. Scandinavian Journal of Rehabilitation Medicine. 1988; 20(3):117-27.

[24] Vera-Garcia FJ, Moreside JM, McGill SM. MVC techniques to normalize trunk muscle EMG in healthy women. Journal of Electromyography and Kinesiology. 2010;20(1):10-6.

[25] Vezina MJ, Hubley-Kozey CL. Muscle activation in therapeutic exercises to improve trunk stability. Archives of Physical Medicine and Rehabilitation. 2000;81(10): 1370-9.

[26] de Oliveira AS, de Morais Carvalho M, de Brum DPC. Activation of the shoulder and arm muscles during axial load exercises on a stable base of support and on a medicine ball. Journal of Electromyography and Kinesiology. 2008;18(3):472-9.

[27] Decker MJ, Hintermeister RA, Faber KJ, et al. Serratus anterior muscle activity during selected rehabilitation exercises. The American Journal of Sports Medicine. 1999;27(6):784-91.

[28] Hardwick DH, Beebe JA, McDonnell MK, et al. A comparison of serratus anterior muscle activation during a wall slide exercise and other traditional exercises. Journal of Orthopaedic \& Sports Physical Therapy. 2006;36(12): 903-10.

[29] Escamilla RF, Yamashiro K, Paulos L, et al. Shoulder muscle activity and function in common shoulder rehabilitation exercises. Sports Medicine. 2009;39(8): 663-85.

[30] Neumann DA. Kinesiology of the musculoskeletal system-e-book: foundations for rehabilitation. Elsevier Health Sciences. 2013.

[31] Kisner C, Colby LA, Borstad J. Therapeutic exercise: foundations and techniques. Fa Davis. 2017.

[32] Page P, Frank C, Lardner R. Assessment and treatment of muscle imbalance: the Janda approach. Journal of Orthopedic \& Sports Physical Therapy. 2011;41(10): 799-800

[33] Ludewig PM, Braman JP. Shoulder impingement: biomechanical considerations in rehabilitation. Man Ther. 2011;16(1):33-9. 